Document downloaded from:

http://hdl.handle.net/10251/121005

This paper must be cited as:

Espinosa-Soria, A.; Pinilla-Cienfuegos, E.; Díaz-Fernández, FJ.; Griol Barres, A.; Martí Sendra, J.; Martínez Abietar, AJ. (2018). Coherent Control of a Plasmonic Nanoantenna Integrated on a Silicon Chip. ACS Photonics. 5(7):2712-2717.

https://doi.org/10.1021/acsphotonics.8b00447

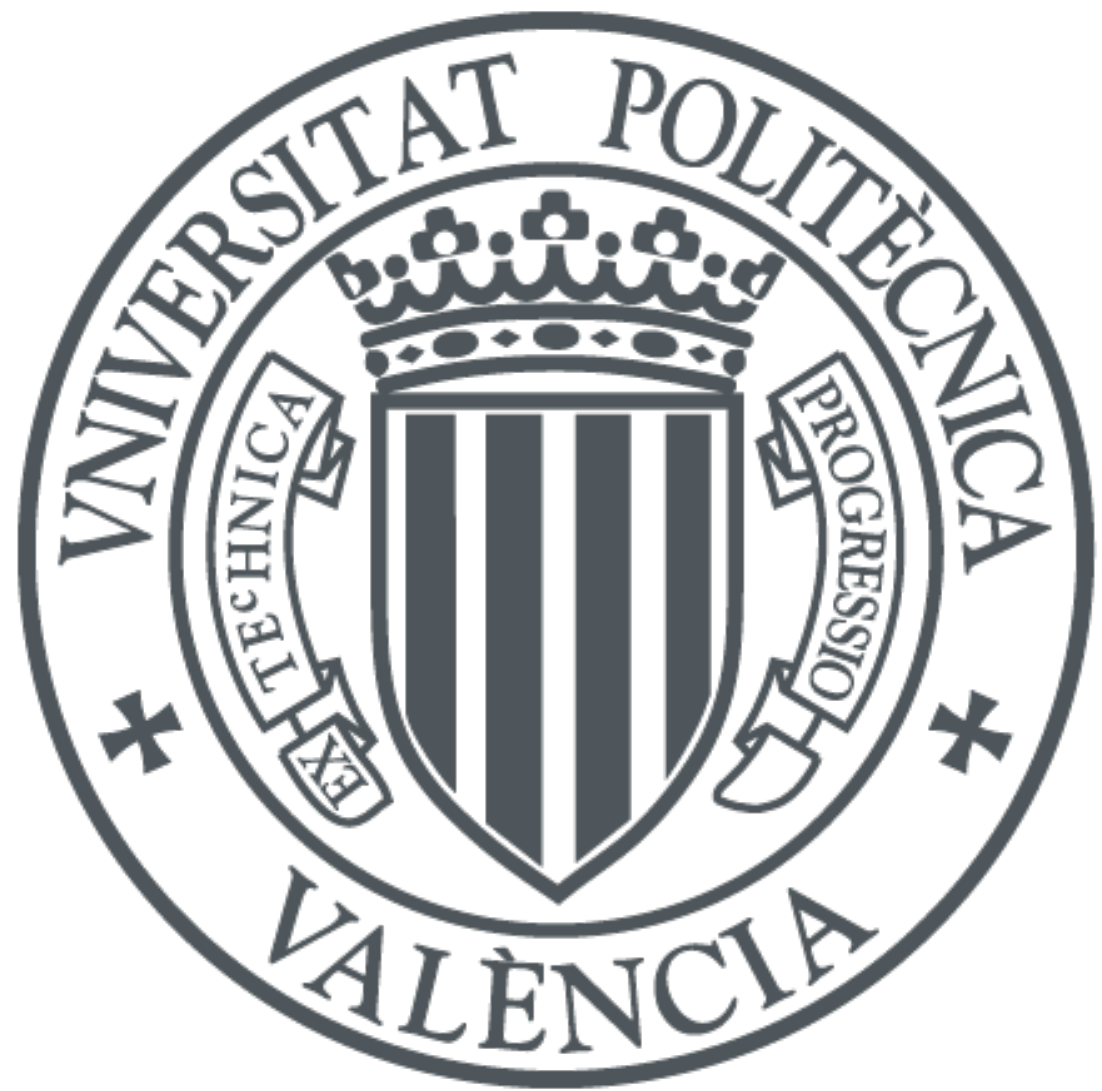

The final publication is available at

http://doi.org/10.1021/acsphotonics.8b00447

Copyright American Chemical Society

Additional Information 


\title{
Coherent control of a plasmonic nanoantenna integrated on a silicon chip
}

\author{
Alba Espinosa-Soria*, Elena Pinilla-Cienfuegos, Francisco J. Díaz-Fernández, Amadeu Griol, Javier \\ Martí and Alejandro Martínez* \\ Nanophotonics Technology Center, Universitat Politècnica de València, Valencia, Spain
}

Keywords: nanoantennas, coherent perfect absorption; silicon photonics; plasmonic resonances; polarization. Supporting Information Placeholder

\begin{abstract}
Illuminating a plasmonic nanoantenna by a set of coherent light beams should tremendously modify its scattering, absorption and polarization properties, thus enabling all-optical dynamic manipulation. However, diffraction inherently makes coherent control of isolated subwavelength-sized nanoantennas highly challenging when illuminated from free-space. Here, we overcome this limitation by placing the nanoantenna at a subwavelength distance of the output facet of silicon waveguides that provide monolithically-defined paths for multibeam coherent illumination. Inspired by coherent perfect absorption (CPA) concepts, we demonstrate experimentally modulation of the nanoantenna scattering by more than one order of magnitude and of the on-chip transmission by $>50 \%$ over a $\sim 200 \mathrm{~nm}$ bandwidth at telecom wavelengths by changing the phase between two counter-directional coherent guided beams. Moreover, we demonstrate coherent synthesis of polarization of the radiated field by illuminating the nanoantenna from orthogonal waveguides. Our finding paves the way towards coherent manipulation of nanoantennas and all-optical processing without nonlinearities in an integrated platform.
\end{abstract}

When a monochromatic light beam illuminates a subwavelength-sized nanoantenna ${ }^{1-3}$, it gets partly either scattered or absorbed, resulting in a certain extinction of the propagating beam (see Fig. 1(a)). Both scattering including its polarization state - and absorption will mainly depend on the shape, size and material composition of the nanoantenna. ${ }^{2}$ However, the situation may become completely different when the nanoantenna is illuminated by a set of coherent beams. Simultaneous illumination of the nanoantenna by two counter-directional coherent light beams should result in either suppressed (Fig. 1(b)) or enhanced (Fig. 1(c)) absorption and scattering depending on the phase difference between the beams. ${ }^{4-10}$ When illuminating absorbing films within this configuration, the regime known as coherent perfect absorption
$(\mathrm{CPA})^{5}$ - for which all light is absorbed - becomes attainable. In the case of subwavelength structures, scattering will be always significant, but controlling the phase of the beams would enable to reach coherent perfect extinction (CPE) - for which no light exists the system - as long as the nanostructure shows a $50 \%$ of extinction for singlebeam illumination. ${ }^{8,9}$ A key aspect of CPA is that a light beam is affected by another light beam without the presence of nonlinear materials. ${ }^{9}$ Therefore, it can be used to perform all-optical intensity modulation, which may bring different advanced functionalities for coherent light processing $^{10}$ without constraints on the maximum speed. ${ }^{11}$ Multipath coherent illumination would also enable to modify the nanoantenna response in other ways. For instance, illuminating a nanoantenna by two orthogonal paths with a certain phase shift between them (Fig. 1(d)) modifies the polarization of the scattered radiation. This scheme enables to tailor at will the polarization response of a given nanoantenna in each spatial direction by controlling the amplitude and phase of the excitation.

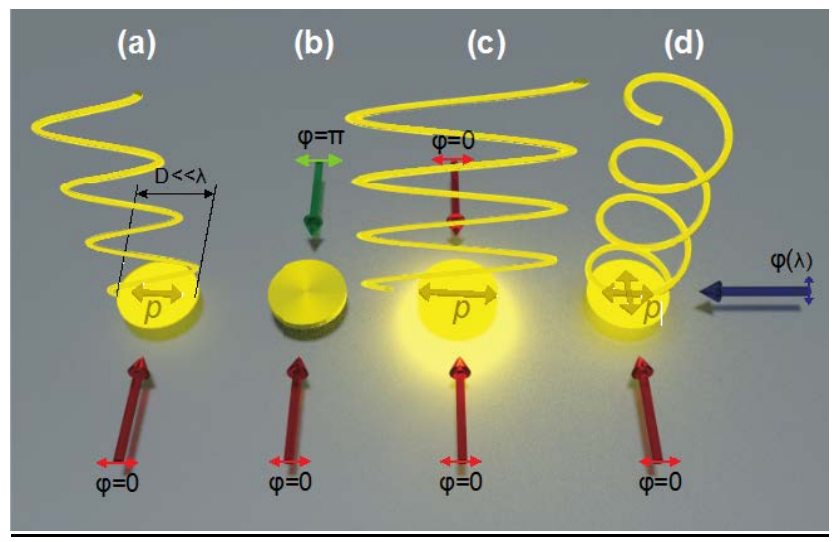

Figure 1. Coherent manipulation of the absorption, scattering and polarization of a plasmonic nanoantenna. (a) Under single-beam illumination, light is partly scattered and absorbed by the nanoantenna depending on the polarization of the input light and the multipolar response of the nanoan- 
tenna. When two coherent, identically-polarized and counter-directional beams illuminate the antenna, scattering and absorption are either suppressed (b) or enhanced (c) depending on the phase shift between pathways, whilst polarization of the scattered light is maintained. When the beams are orthogonal (d), linear superposition enables to modify the polarization of the radiated signal: under a $\pi$ phase difference, circular polarization in the direction of the disk axis becomes feasible. For simplicity we consider that the nanoantenna response is dominated by its in-plane electric dipole resonance, which is a good approximation for thin metallic nanoantennas of subwavelength in-plane dimensions.

Although previous works have measured the scattering and absorption of individual nanostructures by strongly focusing free-space beams, ${ }^{12,13}$ the realization of experiments using multiple beams imposes several challenges. For instance, accurate control of the illumination angles whilst keeping all beams focused in a certain position is required. The system complexity grows as long as more feeding paths are added and it could be unrealizable for controlling multiple nanoantennas. To circumvent this issue, here we propose to embed the nanoantenna in an integrated circuit and use high-index dielectric optical waveguides to illuminate it. ${ }^{14}$ If the nanoantenna is well aligned with the waveguide axis and the distance between the waveguide facet and the nanoantenna is deeply subwavelength, the strong overlap between the guided mode and the nanoantenna resonance will result in highly efficient excitation of the nanoantenna, mimicking the freespace situation with focused beams. Additionally, extra beams can be easily added by placing more waveguides, whose direction can be accurately defined by lithography while their amplitude and phase of the guided light can be controlled either by using on-chip modulators or external mechanisms. Remarkably, this hybrid plasmonic-photonic approach, ${ }^{15}$ which closely resembles the situation in the RF domain where metallic antennas serve as transducer between wireless and wired networks, would naturally enable the simultaneous coherent driving of multiple nanoantennas integrated on a same chip.

\section{RESULTS}

We first consider coherent control of the scattering and absorption of an individual nanoantenna placed in a subwavelength gap (length $g$ ) separating two silicon waveguides (Fig. 2(a)). In particular, we consider a metallic nanostrip supporting an electric dipole resonance along the $y$ axis, which can be efficiently excited by the fundamental TE-like mode of the waveguide. ${ }^{14}$ Two counterpropagating waves are injected through ports A and B to simultaneously illuminate the nanoantenna. This results in both absorption (a) and scattered radiation (partly guided and directed towards the output ports, $s$, being the rest scattered out of the system, $s^{\prime}$ ), which gets maximized when the nanoantenna is resonantly driven. Besides, the system can be characterized by the single-beam waveguide transmission $(t)$ and reflection $(r)$ that account for the discontinuity of the system in the gap when there is no nanoantenna (if $g=0$, then $t=1$ and $r=0$ ). When the nanoantenna is illuminated by equal-amplitude signals having a $\varphi$ phase difference at $x=0$, interference at the nanoantenna position will provoke the out-of-axis scattered intensity $I_{\mathrm{s}}$, to be proportional to $\cos ^{2}(\varphi / 2)$ (see Supp. Information, Section S2). Thus, for $\varphi=0(=\pi)$ there will be constructive (destructive) interference at the nanoantenna and both the scattering and absorption will be maximized (minimized), as can be seen in the numerical simulations shown in Fig. 2(b).
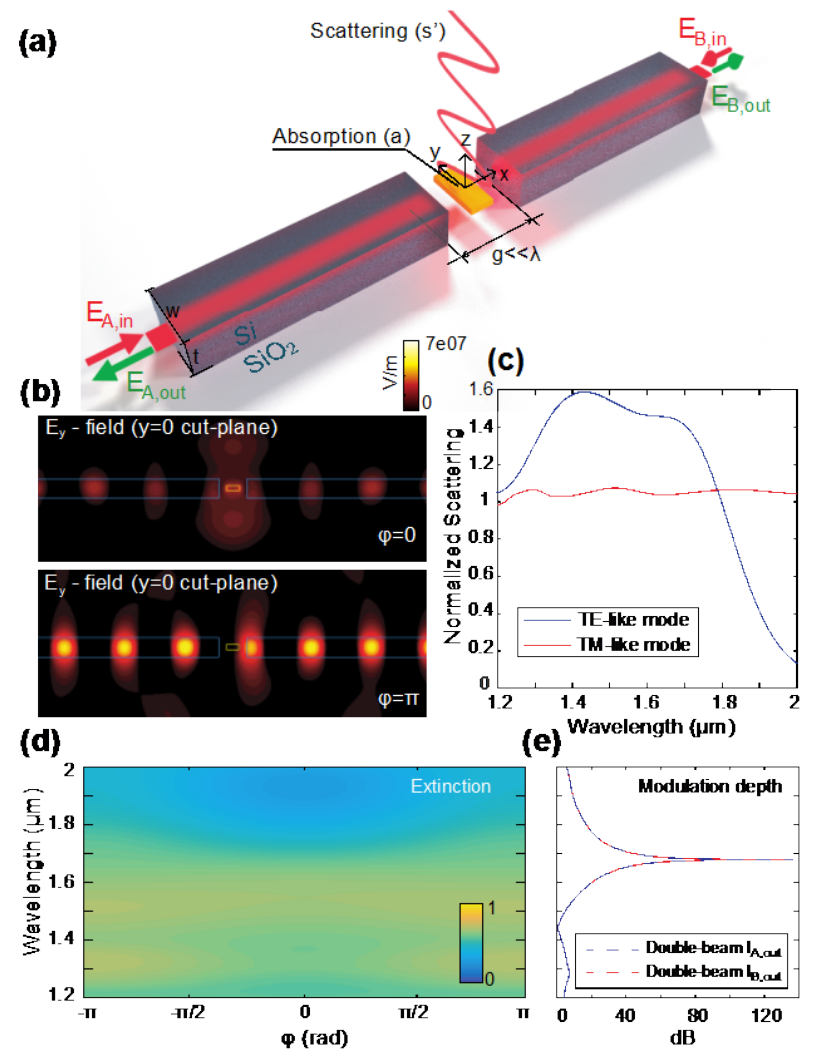

(e)

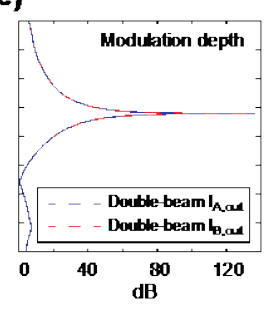

Figure 2. On-chip coherent control of absorption and scattering of a single nanoantenna. (a) Sketch of the proposed structure. (b) Numerical simulation showing the cross-section $(y=0)$ of the electric field (modulus) for enhanced (top) and suppressed (bottom) scattering $(\lambda=1,550 \mathrm{~nm})$. See also the time evolution in Supporting Movies S1 and S2. (c) Simulated normal scattering spectra for TE-like and TM-like single-beam illumination with nanoantenna normalized respect to the system without nanoantenna. (d) Simulated joint extinction $E$ - defined as the normalized power which does not exit the system as a guided wave - as a function of both the wavelength $\lambda$ and the phase difference between counterpropagating signals. (e) Maximum transmission modulation depth $M_{\mathrm{t}}$ as a function of the wavelength for transmission paths A and B. At each wavelength the phase difference that maximizes and minimizes the output intensity are chosen. Values of $M_{\mathrm{t}}$ of tens of $\mathrm{dB}$, large enough for all-optical processing, are achieved at $\lambda \approx 1,690 \mathrm{~nm}$ for one of the two paths, even though there is not CPE. In the simulations, we have considered a 125x320x40 nm gold nanostrip, placed in the 
center of a $g=300 \mathrm{~nm}$ gap separating two silicon waveguides with $400 \times 250 \mathrm{~nm}^{2}$ cross-section. The system is covered by silica. More details about the numerical simulations are given in Methods.

When the system has perfect mirror symmetry with respect to $x=0$, and assuming that the nanoantenna length along the $x$-axis is much smaller than the wavelength, both nanoantenna scattering and absorption should be completely cancelled for $\varphi= \pm \pi$ since the transverse electric field at the nanoantenna position vanishes. It should be noticed too that breaking the translational symmetry of the waveguides because of the gap also produces out-ofaxis scattering even when the nanoantenna is not present. However, numerical simulations (see Fig. 2(c)) show that the normal scattering will be dominated by the nanoantenna response when using the fundamental TE-like mode. For the TM-like mode the nanoantenna resonance is not excited and the waveguide-breaking dominates the scattering.

(a)
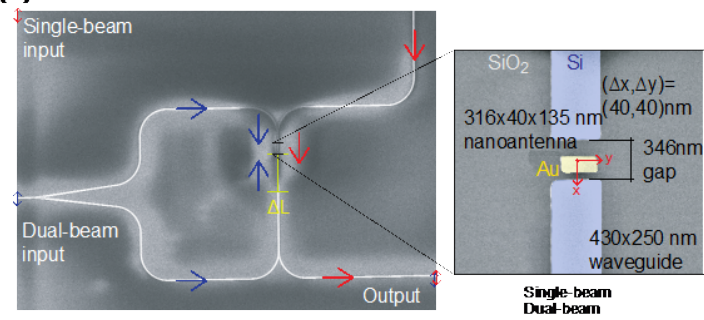

(b)

MUMERICAL EXPERIMENTAL

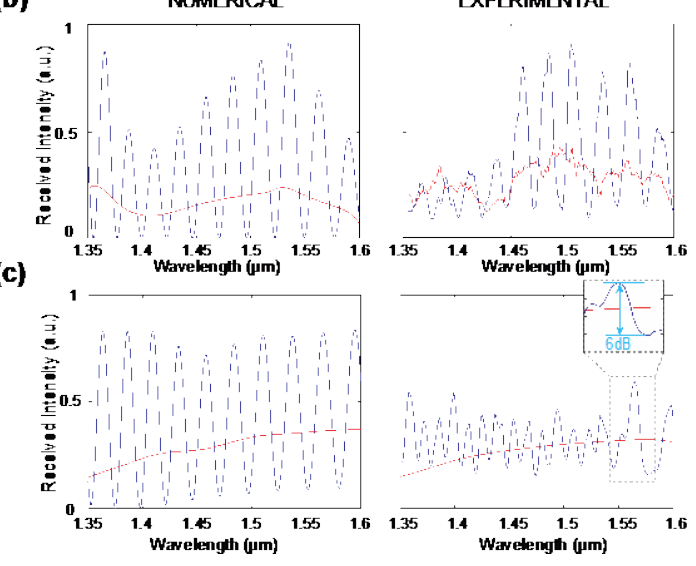

Figure 3. Far-field experimental results. (a) SEM images of the sample configuration. The image on the left shows the configuration of the sample, where both single-beam (red path) and dual-beam (blue path) excitation can be achieved for the same nanoantenna. The false-color image on the right shows the dimensions of the fabricated nanoantenna, $135 \times 316 \times 40 \mathrm{~nm}$, with an in-plane misalignment from the gap center of $(\Delta x, \Delta y)=(40,40) \mathrm{nm}$. The path length difference between feeding waveguides is $\Delta L=21 \mu \mathrm{m}$. (b-c) Numerical and experimental results of the fabricated sample, both for scattering (b) and transmission (c) using the waveguide fundamental TE-like mode. The response to the single-beam (dual-beam) excitation is depicted in red (blue).

Interference at the nanoantenna position also modulates the on-chip transmission and reflection paths. Although the output intensity (for example, $I_{\mathrm{B}, \mathrm{out}}$ ) shows a more intricate behavior than the scattering (see Supp. Information, Section S.3), under certain conditions a cosinelike (for $r=t$ ) or sine-like (for $2 s=-r-t$ ) response with respect to $\varphi$ can still be attained. Such conditions also lead to CPE for $\varphi= \pm \pi$ or $\varphi=0$, respectively, which however is not achieved in our experimental system (see more details in Section S.4). As shown in Fig. 2(d), the calculated joint extinction $E$ reaches maximum values of $E \approx 0.7$ at $\lambda \approx$ $1,330 \mathrm{~nm}$ and $\varphi \approx \pi$, which means that still $\sim 30 \%$ of the power is exiting the system as guided waves. Noticeably, all-optical coherent operations may require large on-chip modulation depth $\left(M_{\mathrm{t}}\right)$ - defined as the ratio between the maximum and minimum output intensity achievable at a certain wavelength - only along one of the system paths, ${ }^{10}$ which enormously relaxes the constraint on the $\varphi$ values cancelling the output and can be well satisfied in our system (Fig. 2(e)).

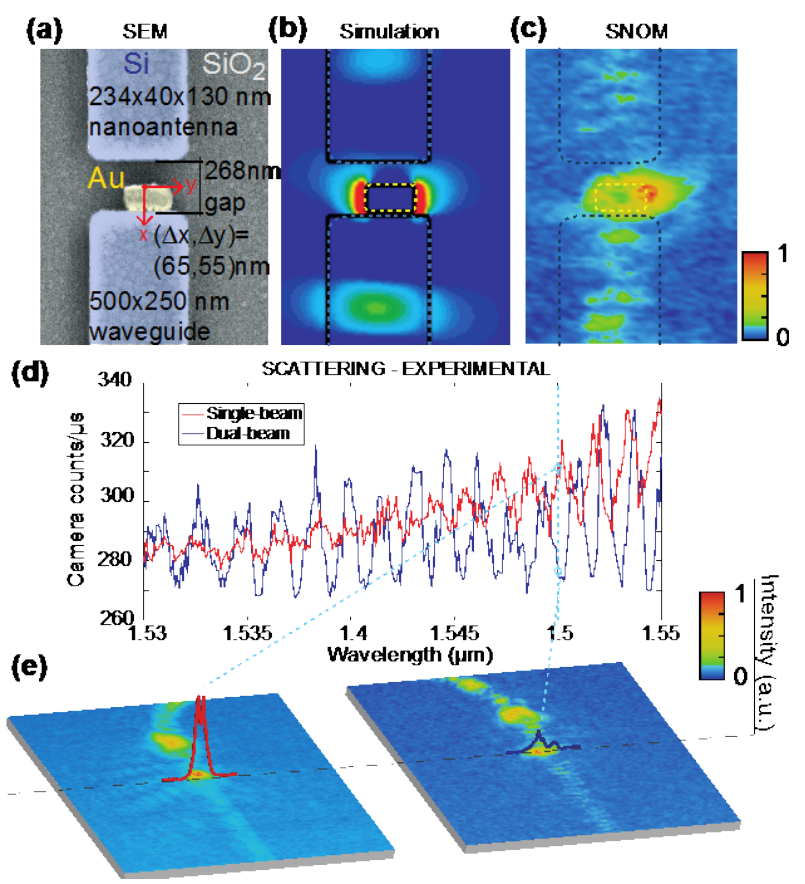

Figure 4. Near-field experimental results. We used a second sample with the same circuit as in Fig. 3(a) but with $\Delta L=300$ $\mu \mathrm{m}$ and $(\Delta x, \Delta y)=(65,55) \mathrm{nm}$. (a) SEM image, (b) simulations and (c) near-field image (see details in Methods) at $\lambda=$ $1,550 \mathrm{~nm}$. (d) Far-field normal scattering for both singlebeam and dual-beam illuminations. (e) 2D maps and crosssection at the nanoantenna position from near-field measurements on a $20 \times 20 \mu \mathrm{m}$ region for single-beam and dual-beam illumination at the wavelength highlighted with the vertical dashed line in (d).

Counter-propagating signals having equal amplitude but phase shifted by $\varphi$ are achieved in an on-chip configuration by splitting a laser-driven input waveguide into two different waveguides that are then bent to finally illuminate the nanoantenna in opposite directions (see Fig. $3(\mathrm{a})$ ). If there is a length difference $\Delta L$ between both paths and the effective index of the waveguide mode is $n$ (see 
Supp. Information, Section S.6), then $\varphi=2 \pi n \Delta L / \lambda$. Importantly, the photonic circuit in Fig. 3(a) also enables single-beam illumination measurements (red path in Fig. 3(a)). The nanoantenna is embedded in the gap, though there are small misalignments with respect to the waveguide axis and the $x=0$ plane as a result of inaccuracies in the second lithography step (fabrication details are described in Methods), which should slightly reduce the excitation efficiency ${ }^{16}$ Figure 3(b) depicts the experimental top-view scattering and on-chip transmitted intensity (see also Methods) for $\Delta L=21 \mu \mathrm{m}$, which are in good agreement with the numerical simulations (also depicted). As expected, for dual-beam illumination the scattering shows a large modulation (about one order of magnitude in a $\sim 200 \mathrm{~nm}$ bandwidth region) with respect to the singlebeam illumination, which is a clear signature of the coherent control of the nanoantenna. Unlike in the ideal case (perfect mirror symmetry with respect to $x=0$ ), where scattering completely cancels out for destructive interference, the measured scattering minima are not zero because of the alignment inaccuracies. A large modulation in transmission $(\approx 75 \%$-or $6 \mathrm{~dB}$ - in some spectral regions, as shown in the inset) is also observed in experiments. This greatly exceeds previous approaches in which the nanoantenna is placed on top of the waveguide, for which the intensity modulation is always lower than $20 \%$ even when using silicon nitride waveguides with poorer mode confinement. ${ }^{17,18}$ Discrepancies between simulations and experiments, more evident at short wavelengths, could be due to excitation of higher-order plasmonic modes in the nanoantenna as well inter-modal conversion, ${ }^{19}$ which are not considered in our model. We plan to conduct future work to study such phenomena.

We fabricated another sample with $\Delta L=300 \mu \mathrm{m}$ and performed near-field measurements in addition to the farfield scattering (see Fig. 4). Suppression of the nanoantenna near-field enhancement for dual-beam illumination at a destructive-interference wavelength is clearly seen. Near-field measurements also confirm the nanoantennagap misalignment observed in the SEM images, as well as the different amount of light in each waveguide because of the interference.

Our approach also enables extra degrees of control of the nanoantenna properties. In particular, by using orthogonal illumination paths (see Fig. 5(a)), the polarization of the emitted radiation can be tailored in a coherent way. Assuming a nanoantenna with rotational symmetry around the $z$ axis which is driven using the TE-like mode of the waveguides with complex amplitudes $E_{A, i n}$ and $E_{B, i n}$, the nanoantenna radiation in the normal direction will be proportional to $E_{A, i n} \hat{x}+E_{B, i n} \hat{y}$, so any polarization state can be synthesized (see Supporting Information, Section S.8). This includes circular polarization even though the nanoantenna is not chiral, which is a clear advantage of the coherent feeding. In the fabricated device, we used an Au disk nanoantenna (inset in Fig. 5(a)) in a photonic circuit similar to that in Fig. 3(a). Figure 5(b) shows the polarization of the radiation - in terms of its Stokes parameters ${ }^{20}$ - emitted in the normal direction as a function of the wavelength, showing a remarkable agreement with the simulations.
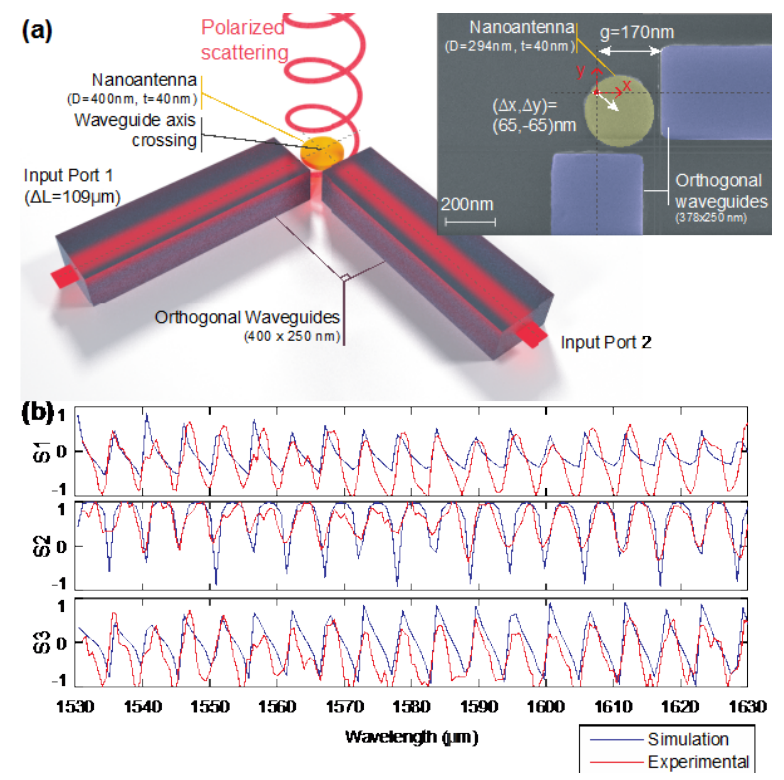

Figure 5. Coherent polarization synthesis. (a) Sketch and SEM image (inset) of the structure, in which two orthogonal waveguides illuminate an Au disk nanoantenna. The orthogonal waveguides cross section is $378 \times 250 \mathrm{~nm}$, separated 170 $\mathrm{nm}$ from the crossing of the waveguides axis. The nanodisk diameter is $294 \mathrm{~nm}$ with $40 \mathrm{~nm}$ thickness, and its center is displaced $(\Delta x, \Delta y)=(65 \mathrm{~nm},-65 \mathrm{~nm})$ from the axis center. The path length difference between feeding waveguides is $\Delta L=109 \mu \mathrm{m}$. (b) Experimental (red) and numerical (blue) results of the state of polarization of the normally-scattered radiation at different wavelengths. The state of polarization is depicted using the Stokes parameters $S_{1}, S_{2}$ and $S_{3}$.

More polarization states could be obtained by changing the amplitude of one of the feeding paths. This element for polarization synthesis complements recent approaches for polarization measurement ${ }^{20}$ or splitting ${ }^{21}$ using plasmonic nanoantennas integrated with silicon waveguides, leading to a set of tools for polarization manipulation in integrated circuits mediated by nanoantennas.

\section{DISCUSSION}

Our coherent approach closely mimics the free-space near-field illumination but in an integrated approach. Embedding the nanoantenna in the gap provides a strong overlapping with the guided modes and a huge intensity modulation contrast in both scattering and transmission. Specifically, the $50 \%$ single-beam extinction required for $\mathrm{CPE}^{8}$ cannot be achieved with a single nanoantenna placed on top of a waveguide. ${ }^{17-18,22}$ Only by placing a set of nanoantennas on top of the waveguide would result in large single-beam extinction and, ultimately, CPE. ${ }^{23-25}$ Modulation of the scattering of a nanoantenna placed on 
top of a waveguide was also recently shown experimentally, being the interaction enhanced by using dielectric resonant cavities. ${ }^{26}$ However, the use of fully-illuminated embedded plasmonic nanostructures provides the right platform for completely controlling the nanoantenna scattering as well as achieving large intensity modulation of the guided light in a subwavelength-size foot-print. In addition, coherent interference using orthogonal paths also provides a versatile tool for polarization synthesis.

In our samples, the phase difference between illuminating beams was achieved via waveguide dispersion for simplicity. In practical applications, such a phase difference could be modified dynamically by electro-optical ${ }^{27}$ or all-optical modulation ${ }^{26}$ of the waveguide modes. That would allow coherent manipulation of the nanoantenna scattering, absorption and polarization of the nanoantenna at speeds well above $1 \mathrm{GHz}$. Although the large scattering of the employed nanoantennas prevents the realization of CPA, we envisage that using nanoantennas tailored to support a non-radiative anapole mode ${ }^{29}$ or via scattering cancellation techniques, ${ }^{30}$ should allow for single-particle CPA, thus paving the way for a plethora of coherent processing devices ${ }^{10}$ on silicon chips. In contrast, super-scattering nanoantennas ${ }^{31}$ would be desirable for building massive arrays of nanoantennas acting as wireless-towireline transducers in silicon chips.

\section{METHODS}

\section{Numerical simulations}

Numerical simulations were performed using the commercial 3-D full-wave solver CST Microwave Studio, which implements finite integration technique. Hexahedral mesh with 10 cells per wavelength has been used, except in metallic inclusions, where the mesh has been refined up to $\sim 15 \mathrm{~nm}$. Open boundary conditions (perfectly matched layers) were chosen for all external facets. Metallic elements were made of gold, whose optical constants were obtained from ellipsometry measurements of thin films deposited using the same procedure as in the sample fabrication. The refractive indices for silicon and silica were considered to be 3.45 and 1.45 respectively. The whole system (waveguides and nanoantenna) was considered to be surrounded by silica. For normal scattering measurements the nanoantenna was considered as a receiver, with a normally incident plane wave of two orthogonal polarizations ( $x$ and $y$ ), and recording the complex spectrum of the mode amplitude excited in each of the two waveguides. The behavior as an emitting nanoantenna with arbitrary feeding amplitudes was then immediately known by applying superposition and reciprocity.

\section{Far-field measurements}

The experimental setup is depicted in Supporting Figure S8. Coherent light was generated in a tunable laser (SANTEC TSL-210F), covering a wavelength range from $1,350 \mathrm{~nm}$ to $1,600 \mathrm{~nm}$. After passing through a polarization controller that allowed for switching between the TE and TM guided modes, it was coupled to the sample through a lensed fiber. By feeding the chip via the singlebeam (dual-beam) input we measured both the scattering and transmission of the nanoantenna for the single-beam (dual-beam) illumination case. Still, the system allows for performing measurements of the normal scattering as well as the transmitted on-chip intensity. Normal scattering - vertical output light - was measured using an IR camera (Xenics XS-XC117) coupled to the eyepiece of a $4 \mathrm{x}$ microscope (National Stereoscopic Microscopes Zoom model 420 series). The transmission - horizontal output light - was split into two beams in order to simultaneously display the light spot using an IR camera (Indigo Alpha NIR) and measure the output guided power with a power meter (Newport 1930C).

\section{Near-field measurements.}

Scanning Near-field Optical Microscopy (SNOM) measurements were performed with a tailored MultiView 4000 system (Nanonics Imaging Ltd) working in collection mode. A bent fiber tip (Nanonics Imaging Ltd) with a $400 \mathrm{~nm}$ aperture premounted on a tuning-fork working in tapping mode, was used to scan the lithographed sample while it was kept fixed. The sample was laterally illuminated with a lensed fiber connected to a tunable laser (Keysight Agilent Laser, 81940A) and mounted on a 3D stage (More detailed technical description can be found in the Supporting Information, S11).

\section{Fabrication}

Silicon structures were fabricated on standard siliconon-insulator samples from SOITEC wafers with a top silicon layer thickness of $250 \mathrm{~nm}$ and a buried oxide layer thickness of $3 \mu \mathrm{m}$. Patterns were defined by using an electron-beam direct-writing process performed on a coated $100 \mathrm{~nm}$ hydrogen silsesquioxane (HSQ) resist film. The electron-beam exposure, performed with a Raith150 tool, was optimized to reach the required dimensions employing an acceleration voltage of $30 \mathrm{keV}$ and an aperture size of $30 \mu \mathrm{m}$. After developing the HSQ film using tetramethylammonium hydroxide, the resist patterns were transferred into the samples by employing an optimized Inductively Coupled Plasma-Reactive Ion Etching process with fluoride gases. After etching, a 105 nm-thickness $\mathrm{SiO}_{2}$ layer was deposited on the SOI sample by using a plasma enhanced chemical vapor deposition (PECVD) system from Applied Materials. This layer is deposited to center the metallic nanostructure with the optical axis of the waveguide, ensuring maximum interaction with the field within the gap. A second e-beam exposure prior to a metal evaporation and lift-off processes were carried out to define the $40 \mathrm{~nm}$ thickness Au nanoparticle inside the waveguide gaps. A $2 \mathrm{~nm}$ titanium layer were also evaporated to improve gold adhesion. Finally, a micron-thickness silicon dioxide uppercladding was deposited on the SOI sample by using again PECVD in order to ensure homogeneity in the surroundings of the metallic nanostructure. 


\section{ASSOCIATED CONTENT}

\section{Supporting Information.}

Scattering Matrix formalism of the system for coherent control of absorption and scattering, modulation of scattering, absorption, extinction, transmission and reflection, coherent perfect extinction and transmission, waveguide dispersion, supporting simulation results, coherent synthesis of the polarization, numerical modelling of the disk nanoantenna, farfield and near-field experimental measurements (PDF).

Movies S1-S2: Cross-section of the field modulus for enhanced (S1) and suppressed (S2) scattering at $\lambda=1,550 \mathrm{~nm}$ (AVI).

\section{AUTHOR INFORMATION}

\section{Corresponding Author}

*E-mail: amartinez@ntc.upv.es

*E-mail: alesso@ntc.upv.es

\section{Notes}

The authors declare no competing financial interest.

\section{ACKNOWLEDGMENTS}

The authors acknowledge support from the Spanish Ministry of Economy and Competiveness (MINECO): A.E.-S. under grant BES-2015-073146, E.P.-C under grant FJCI-201527228, F.J.D.-F. under grant TEC2015-63838-C3-1-R, A. M. under grants TEC2014-51902-C2-1-R and TEC201461906-EXP.

\section{REFERENCES}

1. Mühlschlegel, P.; Eisler, H.-J.; Martin, O. J. F.; Hecht, B.; Pohl, D. W. Resonant optical antennas. Science 2005, 308, 1607-1609.

2. Novotny, L.; van Hulst, N. F. Antennas for light. Nat. Photonics 2011, 5, 83-90.

3. Koenderink, A. F.; Alù, A.; Polman, A. Nanophotonics: shrinking light-based technology. Science 2015, 348, 516521.

4. Chong, Y. D.; Ge, L.; Cao, H.; Stone, A. D. Coherent Perfect Absorbers: Time-Reversed Lasers. Phys. Rev. Lett. 2010, 105, 053901.

5. Wan, W. et al. Time-Reversed Lasing and Interferometric Control of Absorption. Science 2011, 331, 889.

6. Zanotto, S. et al. Perfect energy-feeding into strongly coupled systems and interferometric control of polariton absorption. Nat. Phys. 2014, 10, 830-834.

7. Pirruccio, G. et al. Coherent Control of the Optical Absorption in a Plasmonic Lattice Coupled to a Luminescent Layer. Phys. Rev. Lett. 2016, 116, 103002.

8. Baranov, D. G. et al. Coherent perfect absorbers: linear control of light with light. Nat. Rev. Mat. 2017, 2, 17064.

9. Z Zhang, J.; MacDonald, K. F.; Zheludev, N. I. Controlling light-with-light without nonlinearity. Light Sci. Appl. 2012, 1, e8.

10. Fang, X.; MacDonald, K. F.; Zheludev, N. I. Controlling light with light using coherent metadevices: all-optical transistor, summator and invertor. Light Sci. Appl. 2015, 4, e29.

11. Fang, X. et al. Ultrafast all-optical switching via coherent modulation of metamaterial absorption, Appl. Phys. Lett. 2014, 104, 141102.
12. Husnik, M. et al. Absolute extinction cross-section of individual magnetic split-ring resonators. Nature Photon. 2008, 2, 614.

13. Fan, P.; Yu, Z.; Fan, S; Brongersma, M. L. Optical Fano resonance of an individual semiconductor nanostructure. Nature Mater. 2014, 13, 471-475.

14. Espinosa-Soria, A.; Griol, A.; Martínez, A. Experimental measurement of plasmonic nanostructures embedded in silicon waveguide gaps. Opt. Express 2016, $24,9592$.

15. Rodriguez-Fortuño, F. J.; Espinosa-Soria, A.; Martinez, A. Exploiting metamaterials, plasmonics and nanoantennas concepts in silicon Photonics. J. Opt. 2016, 18, 123001.

16. Castro-Lopez, M.; de Sousa, N.; Garcia-Martin, A.; Gardes, F. Y.; Sapienza, R. Scattering of a plasmonic nanoantenna embedded in a silicon waveguide. Opt. Express 2015, 23, 28108.

17. Chamanzar, M.; Xia, Z.; Yegnanarayanan, S.; Adibi, A. Hybrid integrated plasmonic-photonic waveguides for onchip localized surface plasmon resonance (LSPR) sensing and spectroscopy. Opt. Express 2013, 21, 32086.

18. Peyskens, F. et al. Bright and dark plasmon resonances of nanoplasmonic antennas evanescently coupled with a silicon nitride waveguide. Opt. Express 2015, 23, 3088.

19. Tellez-Limon, R.; Bahari, B.; Hsu, L.; Park, J.-H.; Kodigala, A.; Kanté, B. Integrated metaphotonics: symmetries and confined excitation of LSP resonances in a single metallic nanoparticle. Opt. Express 2016, 24, 13875.

20. Espinosa-Soria, A.; Rodríguez-Fortuño, F. J.; Griol, A.; Martínez, A. On-chip optimal Stokes nanopolarimetry based on spin-orbit interaction of light. Nano Lett. 2017, 17, 3139.

21. Guo, R.; Decker, M.; Setzpfandt, F.; Gai, X.; Choi, D.-Y.; Kiselev, R.; Chipouline, A.; Staude, I.; Pertsch, T.; Neshev, D. N.; Kivshar, Y. S. High-bit rate ultra-compact light routing with mode selective on-chip nanoantennas. Sci. Adv. 2017, 3, e1700007.

22. Cuadra, J. et al. Hybrid dielectric waveguide spectroscopy of individual plasmonic nanoparticles. AIP Adv. 2017, 7, 075207.

23. Bruck, R.; Muskens, O. L. Plasmonic nanoantennas as integrated coherent perfect absorbers on SOI waveguides for modulators and all-optical switches. Opt. Express 2013, 21, 27652-27661.

24. Wong, Z. J. et al. Lasing and anti-lasing in a single cavity. Nat. Photonics 2016, 10, 796.

25. Zhao, H. et al. Metawaveguide for Asymmetric Interferometric Light-Light Switching. Phys. Rev. Lett. 2016, 117, 19390.

26. Chen, B. et al. Hybrid Photon-Plasmon Coupling and Ultrafast Control of Nanoantennas on a Silicon Photonic Chip, Nano Lett. 2018, 18, 610-617.

27. Brimont, A. et al. High speed silicon electro-optical modulators enhanced via slow light propagation. Opt. Express 2011, 19, 20876-20885.

28. Martínez, A. et al., Ultrafast All-Optical Switching in a Silicon-Nanocrystal-Based Silicon Slot Waveguide at Telecom Wavelengths. Nano Lett. 2010, 10(4), 1506-1511.

29. Miroshnichenko, A. E. et al. Nonradiating anapole modes in dielectric nanoparticles. Nat. Commun. 2015, 6, 8069.

30. Alù, A.; Engheta, N. Achieving transparency with plasmonic and metamaterial coatings. Phys. Rev. E 2005, 72, 01662.

31. Ruan, Z.; Shanhui Fan, S. Superscattering of Light from Subwavelength Nanostructures. Phys. Rev. Lett. 2010, 105, 013901. 
Insert Table of Contents artwork here

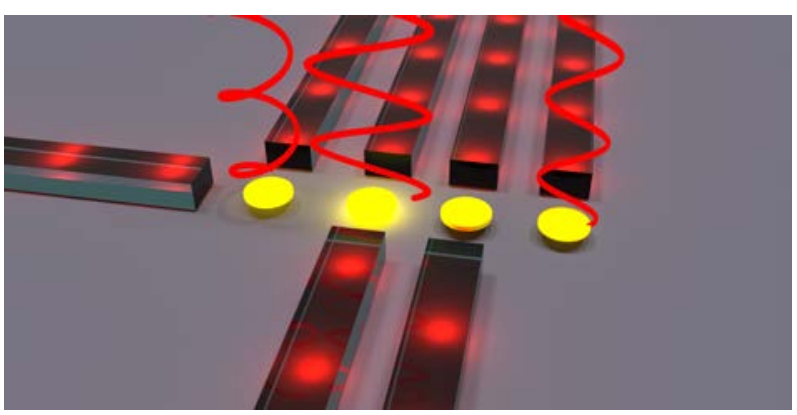

